\title{
Ustilago avenae
}

National Cancer Institute

\section{Source}

National Cancer Institute. Ustilago avenae. NCI Thesaurus. Code C87521.

A species of smut fungi in the phylum Basidiomycota. This species causes loose smut of oats. 